\section{Relation of Helicobacter pylori infection and multiple sclerosis in Iranian patients}

\author{
Nafiseh Mohebi, Mansoureh Mamarabadi, \\ Mehdi Moghaddasi \\ Department of Neurology, Rasool-e- \\ Akram Hospital, Tehran University \\ of Medical Sciences, Tehran, Iran
}

\section{Abstract}

Multiple sclerosis (MS) is the most prevalent central nervous system demyelinating disease. There are known risk factors for MS. However, there is uncertainty in its protective factors. Few studies have demonstrated that some chronic infections may have protective effects on this disease. We carried this study to investigate the relation between Helicobacter pylori (HP) infection and the prevalence and severity of MS. In this case-control study, 163 MS patients and 150 sex- and age- matched controls were included. Blood samples for IgG and IgM anti HP antibodies were collected from all individuals. Also, the Expanded Disability Status Scale (EDSS) was used to evaluate the MS patients. Suitable statistical analysis was applied. A Significant difference was observed in seropositivity between these two groups $(\mathrm{P}<0.001)$ but no significant difference was seen in seropositivity between conventional and opticospinal MS $(\mathrm{P}=0.522)$. No significant difference was observed in seropositivity among ages $(\mathrm{P}=0.075)$ and between genders $(\mathrm{P}=0.204)$. A significant difference was seen in EDSS value between seropositive and seronegative patients $(\mathrm{P}=0.017)$. We concluded that patients with HP infection had lower incidence of multiple sclerosis and MS patients with HP infection showed lower neurologic complications, which can demonstrate that HP infection may have a protective influence on MS pathogenesis.

\section{Introduction}

Multiple sclerosis (MS), as a juvenile disabling disorder, is the most prevalent central nervous system inflammatory and demyelinating disease. ${ }^{1}$ Nearly all researchers believe that focal lymphocytic infiltration in the brain and spinal cord, which is found in MS, can damage the myelin sheet of the neuron axon, which is made by oligodendrocytes. ${ }^{2}$ In the early stages, MS is characterized by recovering episodes of neurological impairments; however, almost any neurological symptom can appear later with the disease, which often progresses to physical and cognitive disability. Two-thirds of the deaths in MS patients are directly related to the consequences of the disease. ${ }^{3}$ Suicide also has a higher prevalence than in the healthy population, and infections and complications are especially hazardous for the more disabled ones. ${ }^{1}$

Although not considered hereditary, a number of genetic variations have been shown to increase the risk of MS. ${ }^{4}$ However, different environmental factors, both of infectious (Epstein-Barr virus and measles) and noninfectious origin (vitamin D deficiency, low uric acid and cigarette smoking) have been demonstrated as risk factors for MS.5-7 Furthermore, some environmental factors have been suspected to have protective effects for MS. Nowadays, chronic infections such as Helicobacter pylori (HP) have been hypothesized to modulate the immune system to prevent its hyperactivity, which can result in allergies and autoimmune disorders. Few studies in high-incidence countries such as Japan have demonstrated that HP has protective effects on MS. ${ }^{8-10}$

$\mathrm{HP}$ is a micro-aerophilic gram-negative bacterium that is present in more than half of any population. Its prevalence positively correlates with age and is well known in gastro-intestinal ulcers pathophysiology. ${ }^{11}$ Compared to other countries with the same latitude, the Iranian population has higher HP prevalence. Although the incidence of MS is rising in high socio-economic levels and its higher rate affects the younger age group, noting the low mean age of our country, this disease can be a heavy economical and physiological burden. 12 Thus, to determine one of the population aspects of these factors, we aim to find the relation between HP infection and MS in Iran.

\section{Materials and Methods}

\section{Subjects}

All patients with definite MS, diagnosed using the McDonald criteria, 13 in RasoulAkram Hospital for the years 2009 and 2010 were included consecutively in our case-control study. The patients were divided into two categories of MS for further evaluation: conventional and opticospinal (based on revised criteria for NMO). 14 The control group consists of non-MS ambulatory clinical patients, who were matched for age and gender. None of the case or control group individuals have received the standard four-drug therapy or any nonstandard medication for HP or any cytotoxic medicine. The study was approved by ethical committee of Tehran University of Medical Sciences and all patients were informed of the
Correspondence: Mehdi Moghaddasi, Neurology Department, Rasool-e-Akram Hospital, Tehran University of Medical Sciences, Niayesh St., Sattar-Khan Ave., Tehran 144561-3131, Iran. Tel. +98.216.6515001 - Fax: +98.216.6517118 E-mail:moghaddasim@hotmail.com

Key words: Helicobacter pylori, multiple sclerosis, immunological influences, Iranian patients.

Contributions: NM, conception and design of the study, preliminary drafting the article, approved the final version of article; MaM, examination of patients, data gathering and analyzed them, and helped with drafting the manuscript; MeM, main conception and design of the study, checked the data analysis, revised and approved the final version of article and submission the article.

Conflict of interest: the authors declare no potential conflict of interests.

Received for publication: 6 September 2012. Revision received: 28 December 2012. Accepted for publication: 22 March 2013.

This work is licensed under a Creative Commons Attribution NonCommercial 3.0 License (CC BYNC 3.0).

(C) Copyright N. Mohebi et al., 2013 Licensee PAGEPress, Italy

Neurology International 2013; 5:e10 doi:10.4081/ni.2013.e10

study and provided their oral and written consent before participating in the study.

Demographic information from all cases was collected from their medical records and entered to a checklist. All cases and controls underwent Krutzke Expanded Disability Status Scale (EDSS) evaluation. ${ }^{15}$ The EDSS quantifies disability in eight functional systems (pyramidal, sensory, visual etc.) and allows neurologists to assign a functional system score (FSS) in each of these. The result is a number between zero (best function, no deficit due to MS) and 10 (death due to MS).

To find which individuals are infected with HP, seropositivity was used. Blood sample was taken from all patients and centrifuged for 10 minutes. Anti HP IgG antibody and anti HP IgM antibody were measured in the individuals using solid- phase enzyme-linked immunosorbent assay (ELISA) based on the sandwich principle (Immune Biological Laboratories GmBH, Hamburg, Germany). Values of more than $12 \mathrm{U} / \mathrm{mL}$ for both tests were considered as positive. Intra-assay and inter-assay $95 \%$ CI for the IgG kit was $8.5 \%$ and $6.3 \%$ and for the IgM kit was $8.1 \%$ and $11.53 \%$, respectively. Individuals with negative IgM and positive IgG were included in our chronic HP seropositive group. 


\section{Data collection and analysis}

All data were entered into SPSS version 16 (SPSS inc. Massachusetts, USA) and appropriate statistical tests such as Chi-square and $t$ test were performed. Quantitative variables are described as mean \pm SD and qualitative variables as percentages. A P value of less than 0.05 was considered significant.

\section{Results}

At the end of the study, 163 MS patients consisting of 76 (47\%) females and 87 (53\%) males were included. The mean age was $32 \pm 8$ years. Our case group consisted of 150 age- and sexmatched non-MS individuals (Table 1).

From the total 163 MS patients, 76 (46.6\%) were convetional MS (CMS) and the other 87 (53.4\%) were opticospinal MS (OSMS). In addition, 137 (84\%) patients suffered from relapsing-remitting MS and the other 26 (16\%) suffered from secondary progressive MS.

HP seropositivity were seen in 88 (54\%) of the MS group and in 110 (73\%) of the non-MS group (Figure 1). A significant difference was seen in seropositivity between these two groups $(\mathrm{P}<0.001)$, but no significant difference was seen in seropositivity between conventional and opticospinal MS [39 (51\%) vs. 49

Table 1. Comparison between the baseline and clinical characteristics of patients and control group.

\begin{tabular}{lccc} 
Mean age (standard deviation) & $\begin{array}{c}\text { Patients } \\
(\mathrm{n}=163)\end{array}$ & $\begin{array}{c}\text { Control } \\
(\mathbf{n}=150)\end{array}$ & $\mathrm{P}$ \\
Gender (male/female) & $32(8)$ & $30(9)$ & 0.407 \\
\hline Mean body mass index (standard deviation) & $87 / 76$ & $82 / 68$ & 0.72 \\
Mean duration of symptoms (range) & $26.4(4.2)$ & $27.5(4.5)$ & 0.14 \\
\hline Hypertension (yes/no) & $34(12-150)$ & - & - \\
Diabetes mellitus (yes/no) & $53 / 110$ & $30 / 120$ & 0.09 \\
\hline Hypothyroid (yes/no) & $15 / 148$ & $9 / 141$ & 0.282 \\
Smoking (yes/no) & $11 / 152$ & $7 / 143$ & 0.643 \\
\hline
\end{tabular}

\section{Discussion}

MS is a multi-factorial disease. Its direct cause has not yet been disclosed and there are several pathways hypothesized for its etiology in the literature. However, to date, autoimmunity is the most acceptable explanation. 1 There have been several studies regarding increased and decreased risk of this disease. Environmental factors such as viral and bacterial infections can precipitate, worsen or protect patients from MS.5,6 This study was performed to demonstrate the relation between HP infection and MS in our population.

In our study, we found a $1.3: 1$ male- to female- ratio. However, in other similar studies, a high female- to male- ratio (2-3:1) was demonstrated, probably due to hormonal influences. Furthermore, the mean age of our individuals was near 32 years, which is significantly lower than other studies.15,16 It seems

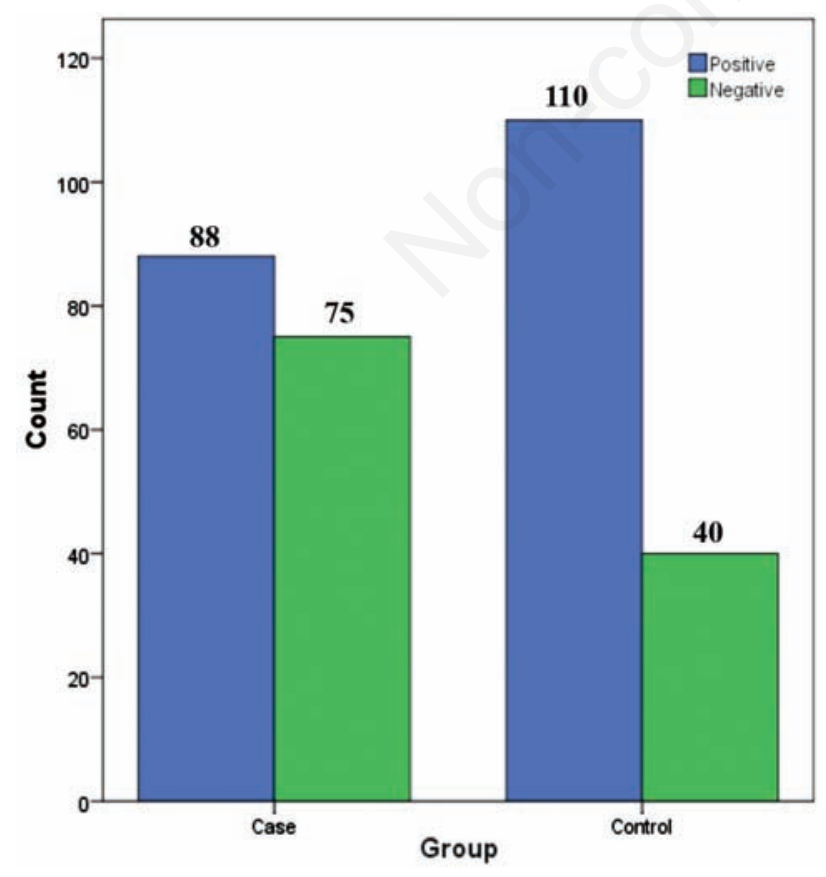

Figure 1. Comparison of anti-Helicobacter pylori test results between multiple sclerosis patients and control group.

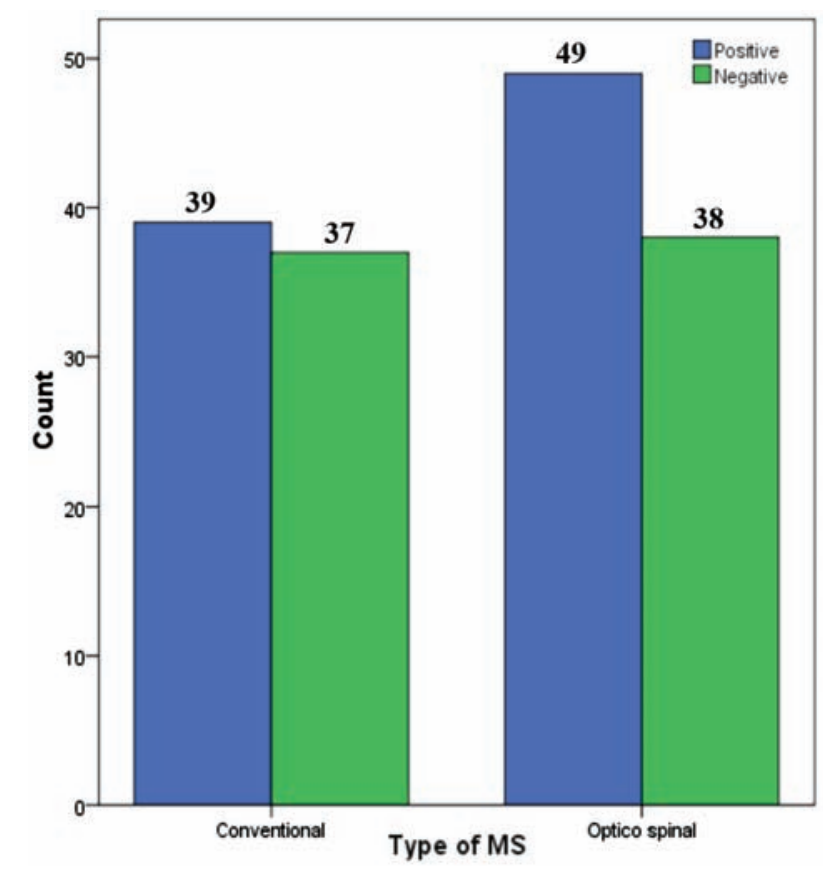

Figure 2. Comparison of anti-Helicobacter pylori test results between conventional and opticospinal multiple sclerosis. 
that the higher prevalence of males in our study was related to the lower age of onset of the disease in our country, perhaps due to ethnical, genetic, or environmental factors. The Iranian population has been categorized into low MS prevalent countries. In the Abedini et al.'s study in 2008, Iran has been categorized into moderate $M S$ prevalent, among other Mediterranean countries such as Egypt and Ukraine, with a ratio of 21.1:100,000.17

This study showed that the prevalence of HP infection was significantly lower in MS patients. However, no significant difference was observed in HP seropositivity in MS and non-MS patients in Wender et al.'s study. ${ }^{18} \mathrm{HP}$ is the most prevalent chronic bacterial infection in humans, as seen in all countries and all ages. Developing countries have more infected population than developed ones, and the prevalence of HP infection in some countries may reach $80 \% .11,19$ There are some explanations why wealthy countries develop more allergic and autoimmune diseases. There are several mechanisms depicting the conceptual framework for the hygiene hypothesis. First, a bacterial infection can influence the balance between cellular T helper 1 and $\mathrm{T}$ helper 2 responses, which is relevant to the development of allergic diseases. Second, infection may be associated with increased productionof interlukin 10, which possesses anti-allergic properties and has been shown to suppress lipopolysacccharide activated eosinophiles in allergic disease models. Third, a bacterial antigen may compete with other environmental antigens responsible for the atopic condition. Fourth, components of bacterial products may also act as superantigens, selectively including activation or deletionof specific T-cell subsets. Fifth, there is emerging evidence that a category of receptors for bacterial components on mononuclear cell membranes, the toll-like receptors, may signal for the production of immunosuppressant cytokines by the mononuclear cells. ${ }^{20}$ Knowing that autoimmunity is one of the most important components in MS, it should be suspected that prior chronic infections can accelerate $\mathrm{T}$ helper type 1 cells and decrease the activation of type $2 \mathrm{~T}$ helpers in these patients.

However, in different types of MS, varying results have been reported. As our study shows, no significant difference was found in HP infected patients between conventional MS (CMS) and opticospinal MS. In a study by Li $e t$ al. in Japan, HP seropositivity was significantly lower in patients with CMS (22.6\%) compared with patients with OSMS (51.9\%) and the normal population $(\mathrm{P}=0.0019$ and $\mathrm{P}=0.0181$, respectively).21 Another study in Japan has also demonstrated higher HP seropositivity in CMS patients. This study has also reported that perhaps HP neutrophil activating protein (HPNAP) may be associated with the pathology in a subgroup of CMS patients, another theory for the HP protective factor. ${ }^{9}$

In our study, HP seropositivity showed a significant inverse correlation with mean EDSS. These findings suggest that $H$. pylori infection is a protective factor against Iranian CMS patients. Same results were also seen in the Li et al. study; suggesting that HP can achieve a protective state even in patients that have already developed MS.

\section{Conclusions}

This study reveals that patients with HP infection have lower incidence or lower complication of MS. These results among other studies can lead to a theory that HP infection may have an immunological or protective influence on MS pathogenesis. More detailed studies with higher sample sizes are recommended.

\section{References}

1. Compston A, Coles A. Multiple sclerosis. Lancet 2010;372:1502-17.

2. Saribas AS, Ozdemir A, Lam C, Safak M. JC virus-induced progressive multifocal leukoencephalopathy. Future Virol 2010;5: 313-23.

3. Compston A, Coles A. Multiple sclerosis. Lancet 2002;359:1221-31

4. Dyment DA, Ebers GC, Sadovnick AD. Genetics of multiple sclerosis. Lancet Neurol 2004;3:104-10.

5. Ascherio A, Munger KL. Environmental risk factors for multiple sclerosis. Part I: the role of infection. Ann Neurol 2007; 61:288-99.

6. Ascherio A, Munger KL. Environmental risk factors for multiple sclerosis. Part II: noninfectious factors. Ann Neurol 2007;61: 504-13.

7. Marrie RA. Environmental risk factors in multiple sclerosis aetiology. Lancet Neurol 2004;3:709-18.

8. Deretzi G, Kountouras J, Gavalas E, et al. Multiple sclerosis and seizures: possible role of Helicobacter pylori. Eur J Neurol 2011;18:e116.

9. Li W, Minohara M, Piao H, et al. Association of anti-Helicobacter pylori neutrophil- activating protein antibody response with anti-aquaporin-4 autoimmunity in Japanese patients with multiple sclerosis and neuromyelitis optica. Mult Scler 2009; 15:1411-21.

10. Pezeshki MZ, Zarrintan S, Zarrintan MH. Helicobacter nanoparticles as a potential treatment of conventional multiple sclerosis. Med Hypotheses 2008;70:122.

11. Mbulaiteye SM, Hisada M, El-Omar EM. Helicobacter pylori associated global gastric cancer burden. Front Biosci 2009; 14:1490-504.

12. Nouraie M, Latifi-Navid S, Rezvan H, et al. Childhood hygienic practice and family education status determine the prevalence of Helicobacter pylori infection in Iran. Helicobacter 2009;14:40-6.

13. McDonald WI, Compston A, Edan G, et al. Recommended diagnostic criteria for multiple sclerosis: guidelines from the International Panel on the diagnosis of multiple sclerosis. Ann Neurol 2001;50:121-7.

14. Wingerchuk DM, Lennon VA, Pittock SJ, et al. Revised diagnostic criteria for neuromyelitis optica. Neurology 2006;66:1485-9.

15. Cottrell DA, Kremenchutzky M, Rice GP, et al. The natural history of multiple sclerosis: a geographically based study 5 : the clinical features and natural history of primary progressive multiple sclerosis. Brain 1999;122:625-39.

16. Scalfari A, Neuhaus A, Degenhardt A, et al. The natural history of multiple sclerosis: a geographically based study 10: relapses and long-term disability. Brain 2010; 133:1914-29.

17. Abedini M, Habibi Saravi R, Zarvani A, Farahmand M. Epidemiologic study of multiple sclerosis. J Mazandaran Univ Med Sci 2008;18:82-6.

18. Wender M. [Prevalence of Helicobacter pylori infection among patients with multiple sclerosis]. Neurol Neurochir Pol 2003;37:45-8. [Article in Polish].

19. Latif AH, Shami SK, Batchoun R, et al. Helicobacter pylori: a Jordanian study. Postgrad Med J 1991;67:994-8.

20. Cremonini F, Gasbarrini A. Atopy, Helicobacter pylori and the hygiene hypothesis. Eur J Gastroenterol Hepatol 2003;15:635-6.

21. Li W, Minohara M, Su JJ, et al. Helicobacter pylori infection is a potential protective factor against conventional multiple sclerosis in the Japanese population. J Neuroimmunol 2007;184:227-31. 